\title{
Triglyceride is strongly associated with nonalcoholic fatty liver disease among markers of hyperlipidemia and diabetes
}

\author{
MINORU TOMIZAWA ${ }^{1}$, YUJI KAWANABE ${ }^{2}$, FUMINOBU SHINOZAKI ${ }^{3}$, SUMIHIKO SATO $^{2}$, \\ YASUFUMI MOTOYOSHI ${ }^{4}$, TAKAO SUGIYAMA ${ }^{5}$, SHIGENORI YAMAMOTO $^{6}$ and MAKOTO SUEISHI ${ }^{5}$ \\ ${ }^{1}$ Department of Gastroenterology, National Hospital Organization Shimoshizu Hospital, Yotsukaido, Chiba 284-0003; \\ ${ }^{2}$ Sato Clinic, Chiba City, Chiba 264-0021; Departments of ${ }^{3}$ Radiology, ${ }^{4}$ Neurology, ${ }^{5}$ Rheumatology and, \\ ${ }^{6}$ Pediatrics, National Hospital Organization Shimoshizu Hospital, Yotsukaido, Chiba 284-0003, Japan
}

Received May 24, 2014; Accepted June 27, 2014

DOI: 10.3892/br.2014.309

\begin{abstract}
The aim of the present study was to reveal the metabolic disorders most commonly associated with nonalcoholic fatty liver disease (NAFLD). Triglyceride (TG), high-density lipoprotein cholesterol (HDL), low-density lipoprotein cholesterol (LDL), blood glucose (BG) and hemoglobin A1c (HbA1c) were analyzed. NAFLD was diagnosed using abdominal ultrasound (US), and TG, HDL, LDL, BG and HbA1c were immediately collected on the same day and subjected to multivariate regression analysis. Stepwise analysis was performed to select the variables that were closely associated with NAFLD. The patients who were positive for the hepatitis B antigen and hepatitis C antibody were excluded from the study. Additionally, the patients who were prescribed prednisolone or methotrexate were excluded from the study as these agents may cause NAFLD or liver toxicity. The study included 168 and 125 patients with and without NAFLD, respectively. TG, BG and HbA1c were strongly correlated with NAFLD. Among these parameters, TG was the strongest predictor of NAFLD $\left(\chi^{2}=9.89, \mathrm{P}=0.0017\right)$. TG was the parameter that was most strongly associated with NAFLD. In conclusion, elevated TG was a marker of NAFLD.
\end{abstract}

\section{Introduction}

Nonalcoholic fatty liver disease (NAFLD) is defined as hepatic steatosis without secondary hepatic fat accumulation, including alcoholic consumption, steatogenic medication or hereditary disorders (1). Nonalcoholic fatty liver (NAFL) and nonalcoholic fatty steatohepatitis (NASH) are the categories of NAFLD. With regards to NASH, NAFL is not associated with injury of hepatocytes (ballooning) (1). The survival rate

Correspondence to: Dr Minoru Tomizawa, Department of Gastroenterology, National Hospital Organization Shimoshizu Hospital, 934-5 Shikawatashi, Yotsukaido, Chiba 284-0003, Japan E-mail: nihminor-cib@umin.ac.jp

Key words: multivariate regression analysis, blood glucose, hemoglobin Alc, receiver operating characteristics in patients with NAFLD is lower compared to the general population standardized-mortality ratio due to the risk of cardiovascular disease and hepatocellular carcinoma (2-4). Therefore, it is recommended that NAFLD is diagnosed and treated (5). Abdominal ultrasound (US) is the simplest and most practical diagnostic imaging modality $(6,7)$. To treat NAFLD efficiently, the target of metabolism for treatment should be known. Body mass index (BMI) is a simple marker of obesity and the index is an independent predictor of NAFLD (8). Weight loss is known to improve NASH (9). However, whether weight loss improves NAFL remains unclear. The therapeutic targets, other than BMI, should be investigated.

NAFLD is associated with metabolic disturbances, including diabetes and hyperlipidemia $(1,10)$. Diabetes is an independent predictor of NAFLD (11) and hyperlipidemia is prevalent among patients with NAFLD (12). Insulin-sensitizing agents have been applied to patients with NAFLD. A large, randomized-controlled trial with metformin did not effectively improve NASH (13). Pioglitazone improves steatosis and inflammation (14), but did not improve fibrosis. Steatosis and inflammation are improved with insulin-sensitizing agents; however, it is difficult to treat fibrosis. Statins are important for the treatment of hyperlipidemia. However, simvastatin is not effective in the treatment of NASH (15). The metabolic disorder parameters are not known with regards to NAFLD. Therefore, the present study aimed to investigate the blood examination parameters that were closely associated with NAFLD. Diabetes and hyperlipidemia were studied as they were expected to indicate the therapeutic target of NAFLD.

\section{Materials and methods}

Inclusion criteria. The patients who underwent abdominal US between April 2013 and November 2013, and had laboratory data that were available on the date of the abdominal US, were included in the study. The patient records were analyzed retrospectively, and were divided into two groups: Patients with NAFLD and those without NAFLD. The study was reviewed by the institutional Ethics Committee and it was determined that the study was not a clinical trial as it was performed as part of daily clinical practice. Patient anonymity was preserved throughout the analysis. 
Table I. Patient characteristics.

\begin{tabular}{|c|c|c|c|c|}
\hline Characteristics & Total & Non-NAFLD & NAFLD & P-value \\
\hline Patient, $\mathrm{n}$ & 293 & 125 & 168 & \\
\hline Male/female, $\mathrm{n}$ & $140 / 153$ & $62 / 63$ & $78 / 90$ & \\
\hline Age, years & $67 \pm 13$ & $63.4 \pm 12.9$ & $69.4 \pm 12.8$ & 0.0005 \\
\hline ALP, IU/1 & $241.7 \pm 91.4$ & $225.1 \pm 81.9$ & $253.0 \pm 63.9$ & 0.0030 \\
\hline AST, IU/1 & $27.5 \pm 16.6$ & $27.5 \pm 15.7$ & $23.4 \pm 10.1$ & $<0.0001$ \\
\hline ALT, IU/1 & $27.6 \pm 25.9$ & $35.0 \pm 34.8$ & $19.0 \pm 11.9$ & $<0.0001$ \\
\hline$\gamma$-GTP, IU/1 & $48.2 \pm 66.6$ & $51.1 \pm 28.7$ & $39.5 \pm 56.0$ & 0.0258 \\
\hline HDL, mg/dl & $59.0 \pm 21.3$ & $42.5 \pm 17.6$ & $63.5 \pm 19.0$ & 0.0002 \\
\hline $\mathrm{LDL}, \mathrm{mg} / \mathrm{dl}$ & $115.7 \pm 28.5$ & $114.2 \pm 35.5$ & $112.7 \pm 23.9$ & 0.0483 \\
\hline $\mathrm{TG}, \mathrm{mg} / \mathrm{dl}$ & $128.2 \pm 75.2$ & $160.8 \pm 129.9$ & $104 \pm 56.8$ & $<0.0001$ \\
\hline $\mathrm{BG}, \mathrm{mg} / \mathrm{dl}$ & $122 \pm 47.5$ & $133.2 \pm 40.8$ & $112.6 \pm 42.7$ & 0.0001 \\
\hline HbA1c, \% & $6.30 \pm 1.06$ & $6.67 \pm 0.93$ & $6.00 \pm 0.91$ & $<0.0001$ \\
\hline
\end{tabular}

Continuous variables are expressed as average \pm standard deviation. NAFLD, nonalcoholic fatty liver disease; ALP, alkaline phosphatase; AST, aspartate aminotransferase; ALT, alanine aminotransferase; $\gamma$-GTP, $\gamma$-glutamyl transpeptidase; HDL, high-density lipoprotein cholesterol; LDL, low-density lipoprotein cholesterol; TG, triglyceride; BG, blood glucose; HbA1c, hemoglobin A1c.

Table II. Multivariate logistic regression analysis.

\begin{tabular}{lcccc}
\hline Variables & $\chi^{2}$ & OR & $95 \%$ CI & P-value \\
\hline HDL & 1.34 & 0.9896 & $0.9713-1.0066$ & 0.2469 \\
LDL & 0.37 & 1.0038 & $1.0160-0.9963$ & 0.5419 \\
TG & 9.89 & 1.0088 & $1.0036-1.0146$ & 0.0017 \\
BG & 2.38 & 1.0081 & $0.9984-1.0194$ & 0.1228 \\
HbA1c & 1.58 & 1.3004 & $0.8650-1.9720$ & 0.2084
\end{tabular}

OR, odds ratio; CI, confidence interval; HDL, high-density lipoprotein cholesterol; LDL, low-density lipoprotein cholesterol; TG, triglyceride; BG, blood glucose; HbA1c, hemoglobin A1c.

Table III. Stepwise analysis of the parameters.

\begin{tabular}{lccc}
\hline Variables & $\begin{array}{c}\text { Partial regression } \\
\text { coefficient }\end{array}$ & $\chi^{2}$ & P-value \\
\hline HDL & 0 & 1.5603 & 0.21162 \\
LDL & 0 & 0.5523 & 0.45738 \\
TG & 0.0103 & 16.3461 & $5.28 \mathrm{e}-5$ \\
BG & 0.0066 & 1.7043 & 0.19173 \\
HbA1c & 0.3189 & 2.4377 & 0.11845 \\
\hline
\end{tabular}

HDL, high-density lipoprotein cholesterol; LDL, low-density lipoprotein cholesterol; TG, triglyceride; BG, blood glucose; HbA1c, hemoglobin A1c.

Exclusion criteria. The patients whose laboratory data were not available on the day of US were excluded. The patients who were positive for hepatitis B surface antigen or hepatitis C antibody were also excluded. Liver cirrhosis, primary biliary cirrhosis, autoimmune hepatitis or high alcohol consumption

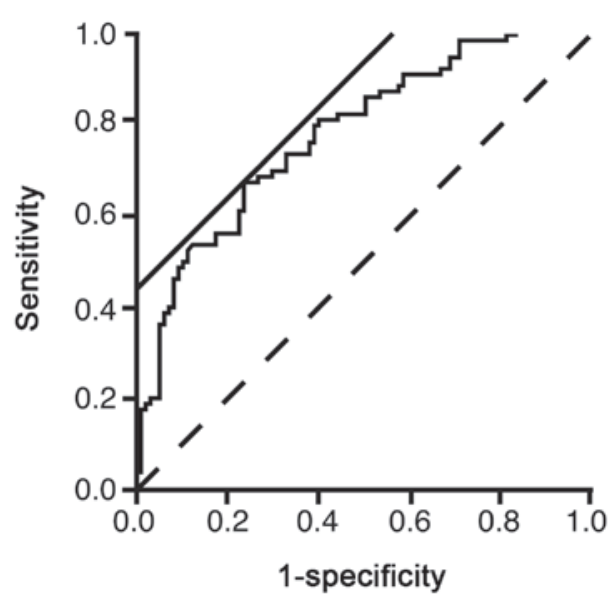

Figure 1. Receiver operator characteristic (ROC) for the prediction of nonalcoholic fatty liver with the logistic model. The ROC curve was illustrated to evaluate the usefulness of the logistic model to predict nonalcoholic fatty liver disease. Solid straight line, a line with a slope of $45^{\circ}$ to calculate the threshold value by the software (JMP 10.0.2); broken line, reference.

were also criteria for patient exclusion due to the possible elevation of their liver enzymes $(16,17)$. The patients with muscular dystrophy or dermatomyositis were excluded due to the possibility of elevated aspartate aminotransferase (AST) or lactate dehydrogenase (LDH) levels. Additionally, patients were excluded from the study if prednisolone was prescribed as it may cause NAFLD (18) and if methotrexate was prescribed as it may cause liver toxicity (19).

Abdominal US. NAFLD was diagnosed using abdominal US, following the standardized criteria $(20,21)$. Briefly, NAFLD was diagnosed when bright liver or hepatorenal echo contrast was observed with abdominal US. Abdominal US was performed by Senior Fellows of the Japan Society of Ultrasonics in Medicine with SSA-700A (Toshiba Medical Systems Corporation, Ohtawara, Japan) using a $3.5-\mathrm{MHz}$ 
curved-array probe. Additionally, abdominal US was performed by Board Certified Fellows of the Japan Society of Ultrasonics in Medicine with SSA-700A using a 5.0-MHz curved-array probe. The investigators were blinded to the clinical and laboratory data.

Laboratory data. The analyzed laboratory data were the levels of alkaline phosphatase (ALP), AST, alanine aminotransferase (ALT), $\gamma$-glutamyl transpeptidase $(\gamma$-GTP), LDH, high-density lipoprotein cholesterol (HDL), low-density lipoprotein cholesterol (LDL), triglyceride (TG), blood glucose (BG) and hemoglobin A1c (HbAlc).

Statistical analysis. One-way analysis of variance was performed for the baseline characteristics. Wald analysis was performed to evaluate the efficiency of each parameter by multivariate regression analysis to predict NAFLD. The receiver operating characteristic (ROC) curve was created to evaluate the predicting performance of the regression model. The threshold value was determined as the highest sensitivity and specificity, and was calculated automatically by the software using the location where a line with a slope of $45^{\circ}$ came into contact with the ROC curve. Stepwise analysis was performed to select variables to predict NAFLD. The statistical software JMP 10.0.2 (SAS Institute, Inc., Cary, NC, USA) was used. $\mathrm{P}<0.05$ was considered to indicate a statistically significant difference.

\section{Results}

Parameters associated with NAFLD. Table I demonstrates the patient characteristics. Age and HDL were significantly lower in patients with NAFLD compared to those without NAFLD $(\mathrm{P}<0.05)$. ALP, AST, ALT, $\gamma$-GTP, LDL, TG, BG and HbA1c were significantly higher in patients with NAFLD compared to those without NAFLD $(\mathrm{P}<0.05)$. Table II demonstrates the logistic regression analysis. TG was the parameter most significantly associated with NAFLD $\left(\chi^{2}=9.89, \mathrm{P}=0.0017\right)$. With the parameters in Table II, the logistic curve was illustrated as an equation: $\ln (\mathrm{P} / 1-\mathrm{P})=-3.8081-0.0104 \times \mathrm{HDL}+0.0038 \times \mathrm{LD}$ $\mathrm{L}+0.0087 \times \mathrm{TG}+0.0081 \times \mathrm{BG}+0.2626 \times \mathrm{HbA} 1 \mathrm{c}(\mathrm{P}<0.0001)$. $\mathrm{P}$ represents the possibility of NAFLD.

The ROC curve was illustrated to evaluate the usefulness of the logistic model to predict NAFLD (Fig. 1). The area under the curve was 0.7739 . The threshold value of $\ln (\mathrm{P} / 1-\mathrm{P})$ was 0.4218 . The sensitivity and specificity of the value were 66.7 and $76.5 \%$, respectively. However, the sensitivity and specificity did not appear to be large enough for using in a clinical setting.

To select the parameters strongly associated with NAFLD, stepwise analysis was performed (Table III). TG, BG and HbA1c were strongly correlated with NAFLD.

\section{Discussion}

Hyperlipidemia is associated with NAFLD. The study by Nakahara et al (22) reported that hyper-LDL cholesterolemia and hypo-HDL cholesterolemia were present in 37.5 and $19.5 \%$ of patients with NAFLD in whom liver biopsy was performed. Hypertriglycemia is most prevalent among patients with NAFLD. Ma et al (23) performed multivariate analysis of
949 retired elderly employees and reported that $\mathrm{HbA1c}$ and TG are independent markers of NAFLD. Sung et al (24) followed healthy workers without NAFLD for 4.4 years to observe the incidence of NAFLD. TG was independently associated with incident NAFLD. These studies indicate that hypertriglycemia is more associated with NAFLD compared to hyper-LDL cholesterolemia and hypo-HDL cholesterolemia. In the present study, TG had the largest $\chi^{2}$ and the smallest P-value (Table II). The data were consistent with the aforementioned previous studies. TG was selected by stepwise analysis as the strongest predictor of NAFLD in comparison with BG and HbA1c (Table III). The data indicated that TG was more closely associated with NAFLD than BG and HbA1c. Therefore, this may indicate that a diet high in carbohydrates may result in increased TG accumulation in hepatocytes $(25,26)$. In conclusion, elevated TG was a marker of NAFLD.

\section{References}

1. Chalasani N, Younossi Z, Lavine JE, et al: The diagnosis and management of non-alcoholic fatty liver disease: practice Guideline by the American Association for the Study of Liver Diseases, American College of Gastroenterology, and the American Gastroenterological Association. Hepatology 55: 2005-2023, 2012.

2. Adams LA, Lymp JF, St Sauver J, et al: The natural history of nonalcoholic fatty liver disease: a population-based cohort study. Gastroenterology 129: 113-121, 2005.

3. Dunn W, Xu R, Wingard DL, et al: Suspected nonalcoholic fatty liver disease and mortality risk in a population-based cohort study. Am J Gastroenterol 103: 2263-2271, 2008.

4. Ascha MS, Hanouneh IA, Lopez R, Tamimi TA, Feldstein AF and Zein NN: The incidence and risk factors of hepatocellular carcinoma in patients with nonalcoholic steatohepatitis. Hepatology 51: 1972-1978, 2010.

5. Chatrath H, Vuppalanchi R and Chalasani N: Dyslipidemia in patients with nonalcoholic fatty liver disease. Semin Liver Dis 32: 22-29, 2012.

6. Musso G, Gambino R, Cassader M and Pagano G: Meta-analysis: natural history of non-alcoholic fatty liver disease (NAFLD) and diagnostic accuracy of non-invasive tests for liver disease severity. Ann Med 43: 617-649, 2011.

7. Festi D, Schiumerini R, Marzi L, et al: Review article: the diagnosis of non-alcoholic fatty liver disease - availability and accuracy of non-invasive methods. Aliment Pharmacol Ther 37: 392-400, 2013.

8. Miyake T, Kumagi T, Hirooka M, et al: Body mass index is the most useful predictive factor for the onset of nonalcoholic fatty liver disease: a community-based retrospective longitudinal cohort study. J Gastroenterol 48: 413-422, 2013.

9. Promrat K, Kleiner DE, Niemeier HM, et al: Randomized controlled trial testing the effects of weight loss on nonalcoholic steatohepatitis. Hepatology 51: 121-129, 2010.

10. Abe N, Honda S and Jahng D: Evaluation of waist circumference cut-off values as a marker for fatty liver among Japanese workers. Saf Health Work 3: 287-293, 2012.

11. Rafiq N, Bai C, Fang Y, et al: Long-term follow-up of patients with nonalcoholic fatty liver. Clin Gastroenterol Hepatol 7: 234-238, 2009.

12. Eguchi Y, Hyogo H, Ono M, et al: Prevalence and associated metabolic factors of nonalcoholic fatty liver disease in the general population from 2009 to 2010 in Japan: a multicenter large retrospective study. J Gastroenterol 47: 586-595, 2012.

13. Haukeland JW, Konopski Z, Eggesbo HB, et al: Metformin in patients with non-alcoholic fatty liver disease: a randomized, controlled trial. Scand J Gastroenterol 44: 853-860, 2009.

14. Vernon G, Baranova A and Younossi ZM: Systematic review: the epidemiology and natural history of non-alcoholic fatty liver disease and non-alcoholic steatohepatitis in adults. Aliment Pharmacol Ther 34: 274-285, 2011.

15. Nelson A, Torres DM, Morgan AE, Fincke C and Harrison SA: A pilot study using simvastatin in the treatment of nonalcoholic steatohepatitis: A randomized placebo-controlled trial. J Clin Gastroenterol 43: 990-994, 2009. 
16. Alvarez F, Berg PA, Bianchi FB, et al: International Autoimmune Hepatitis Group Report: review of criteria for diagnosis of autoimmune hepatitis. J Hepatol 31: 929-938, 1999.

17. Lindor KD, Gershwin ME, Poupon R, Kaplan M, Bergasa NV and Heathcote EJ; American Associaiton for Study of Liver Diseases: Primary biliary cirrhosis. Hepatology 50: 291-308, 2009.

18. Matsumoto T, Yamasaki S, Arakawa A, et al: Exposure to a high total dosage of glucocorticoids produces non-alcoholic steatohepatits. Pathol Int 57: 388-389, 2007.

19. Khan N, Abbas AM, Whang N, Balart LA, Bazzano LA and Kelly TN: Incidence of liver toxicity in inflammatory bowel disease patients treated with methotrexate: a meta-analysis of clinical trials. Inflamm Bowel Dis 18: 359-367, 2012.

20. Sanyal AJ; American Gastroenterological Association: AGA technical review on nonalcoholic fatty liver disease. Gastroenterology 123: 1705-1725, 2002.

21. Bedogni G, Miglioli L, Masutti F, Tiribelli C, Marchesini G and Bellentani S: Prevalence of and risk factors for nonalcoholic fatty liver disease: the Dionysos nutrition and liver study. Hepatology 42: 44-52, 2005.
22. Nakahara T, Hyogo H, Yoneda M, et al; Japan Study Group of Nonalcoholic Fatty Liver Disease (JSG-NAFLD): Type 2 diabetes mellitus is associated with the fibrosis severity in patients with nonalcoholic fatty liver disease in a large retrospective cohor of Japanese patients. J Gastroenterol: Nov 26, 2013 (Epub ahead of print).

23. Ma H, Xu C, Xu L, Yu C, Miao M and Li Y: Independent association of $\mathrm{HbAlc}$ and nonalcoholic fatty liver disease in an elderly Chinese population. BMC Gastroenterol 13: 3, 2013.

24. Sung KC, Kim BS, Cho YK, et al: Predicting incident fatty liver using simple cardio-metabolic risk factors at baseline. BMC Gastroenterol 12: 84, 2012.

25. Lottenberg AM, Afonso Mda S, Lavrador MS, Machado RM and Nakandakare ER: The role of dietary fatty acids in the pathology of metabolic syndrome. J Nutr Biochem 23: 1027-1040, 2012

26. Wiernsperger N: Hepatic function and the cardiometabolic syndrome. Diabetes Metab Syndr Obes 6: 379-388, 2013. 